\title{
Bubble departure diameter at transient heat release
}

\author{
Anatoliy Levin*, and Polina Khan \\ Melentiev Energy Systems Institute SB RAS, 130 Lermontova str., Irkutsk, Russian Federation
}

\begin{abstract}
This paper presents results of the experimental study of the subcooled water boiling on the surface of the cylinder heater. The initial stages of formation of the developed nucleate boiling were investigated. The field temperature of heated liquid layers at the moment of bubble departure was described by numerical modelling at non-stationary condition. A high-speed digital video camera was used to capture the bubble dynamics. The experimental data on the bubble dynamics was compared with Prodanovic's and Song's models. A poor agreement between the predicted departure diameter and the experimental one was obtained for both of the models.
\end{abstract}

\section{Introduction}

Due to the large heat fluxes feasibility, the subcooled liquid boiling has many practical applications. There exist many research results on the subcooled liquid boiling at the constant heat flux or constant heater surface temperature conditions. However, it is still promising to investigate the non-stationary heating, when the surface temperature is gradually increased up to the Leidenfrost point and the heating process goes through the stages of nucleate, transitional, and film boiling. The models predicting bubble departure diameter, nucleation site density, nucleation frequency, and other values are required for the quantitative description of complicated transitional processes.

At present, there exist many models of the bubble evolution based on the various approaches [1-5], which were verified by using the experimental data from such publications as $[1,2]$. In general, these models can be divided into the following groups:

- Mechanistic models, based on the balance of forces applied to a bubble. This approach allows one to predict the bubble behaviour in a vertical channel: liftoff or sliding. However, too many tuning parameters make it difficult to generalize the model.

- Variations of the Zuber model, where the heat exchange and hydrodynamic aspects are expressed in terms of the Jacob and Bond criteria ( $\mathrm{Ja}$ and $\mathrm{Bo}$ numbers). The large number of expressions for the departure diameter and the wall surface temperature clearly indicate that this kind of approach lacks distinctness. The remarkable improvement of the predictive ability of such models was achieved in [3] by introducing the characteristic temperature.

\footnotetext{
*Corresponding author: $\underline{\text { lirt@mail.ru }}$
} 
- Models describing the bubble evolution on the base on the heat balance, including the recent research [4]. The effect of hydrodynamic forces is not included; therefore, such models can predict the maximum diameter without considering the possibility of sliding or lift-off.

In existing publications, all those models were employed under the assumption that the heat flux or heater surface temperature is constant, which predetermined the experimental conditions. Therefore, the dynamics of the superheated liquid layer, as well as the formation and evolution of bubbles in non-stationary heating conditions, is a particular case that requires special experiments and the study of applicability of predictive models.

\section{Results and discussion}

Our experiments with rapidly increasing heating power were conducted on the water flowing in a channel with a cylindrical type 321 stainless steel heater in the center. The heater has the following characteristics: $12 \mathrm{~mm}$ outer diameter, $10 \mathrm{~mm}$ inner diameter, 4 $\mu \mathrm{m}$ roughness. The channel has optically transparent windows and is equipped with the temperature, voltage and pressure measuring probes. Heating power was generated by conducting three-phase rectified electric current through the heater with the pulse duration of $60-300 \mathrm{~ms}$ and the temperature growth rate of $1000-7000 \mathrm{~K} / \mathrm{s}$. The water flowed along the vertical channel upwards with the average velocity of $0.2 \mathrm{~m} / \mathrm{s}$, the inflow pressure of $0.11 \mathrm{MPa}$, and the inflow temperature of 30,60 and $90^{\circ} \mathrm{C}$, corresponding to the subcooling by $12-72 \mathrm{~K}$ with respect to the saturation temperature of $102^{\circ} \mathrm{C}$.

We performed the numerical analysis and obtained the water temperature near the heater surface. We only modelled heating of the homogeneous liquid phase, and, therefore, the temperature field remained adequate until the first bubbles appeared. The axisymmetric 3D model of the steel heater and the water tank was built and verified against the thermocouple data. The laminar flow, the convective and the conductive heat transfer in water, the conductive heat transfer and the joule heat in solid were analyzed using temperature-dependent material properties. The metal temperature at the bottom and at the top was the same as the inflow temperature, taking three values of 30,60 and $90^{\circ} \mathrm{C}$, corresponding to subcooling equal to 72,42 and $12 \mathrm{~K}$. Both in the physical experiment and in the numerical model, the heater temperature was increased due to electric current. The actual voltage history was measured during the physical experiment and used as an input data during the numerical simulation.

According to [2], the maximum diameter can be predicted as follows

$$
D_{m}=\frac{\rho_{L} \alpha_{L}^{2}}{\sigma}\left[\mathrm{A} J a^{b} \theta^{\mathrm{c}}\left(\frac{\rho_{L}}{\rho_{V}}\right)^{d} Q^{e}\right]
$$

where $J a=\frac{\rho_{L} C_{p L}\left(T_{w}-T_{s}\right)}{\rho_{V} r}, \theta=\frac{T_{w}-T_{0}}{T_{w}-T_{s}}, Q=\frac{q}{\rho_{L} v r}, \rho$ is the density, $\alpha$ is the thermal diffusivity, $\sigma$ is the surface tension, $C_{p}$ is the heat capacitance at constant pressure, $T$ is the temperature, $r$ is the latent heat, $q$ is the heat flow rate, $v$ is the liquid velocity, index $L$ referes to the liquid phase, $V$ - to the vapor phase, temperature index $w$ refers to the heater wall, 0 - to the liquid bulk, $s$ - to saturation condition. Figure 1 represents the application of expression (1) to the present data. The calculation results for the coefficients $A, b, c, d, e$, given in [2], were compared with those resulted from our experiment

$$
A=236.75 ; b=2.59 ; c=-0.35 ; d=-1.91 ; e=-1.68 \text {. }
$$




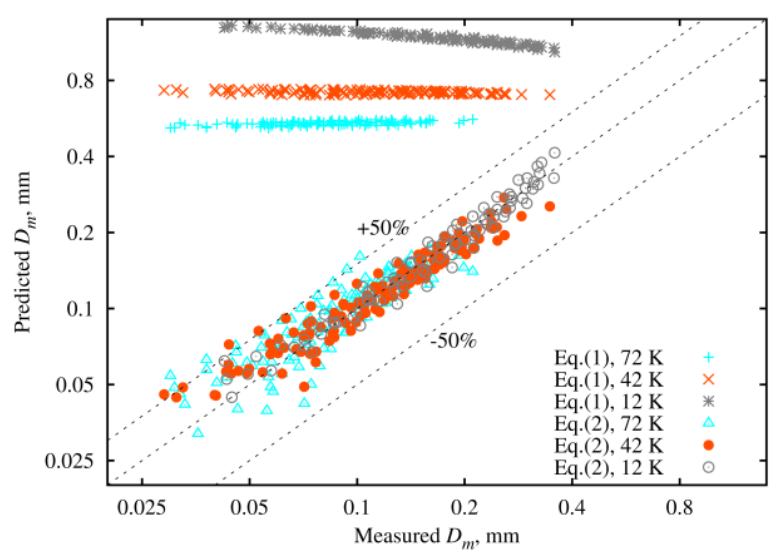

Fig. 1. Results predicted by Prodanovic's correlation against the present data on maximum diameter, obtained using initial and present coefficients.

The difference of correlations in Fig. 1 can be explained by the bigger maximum diameter values (about $1 \mathrm{~mm}$ ) in [2] for the same wall temperatures and heat flow rates, which is typical for the stationary heat release conditions.

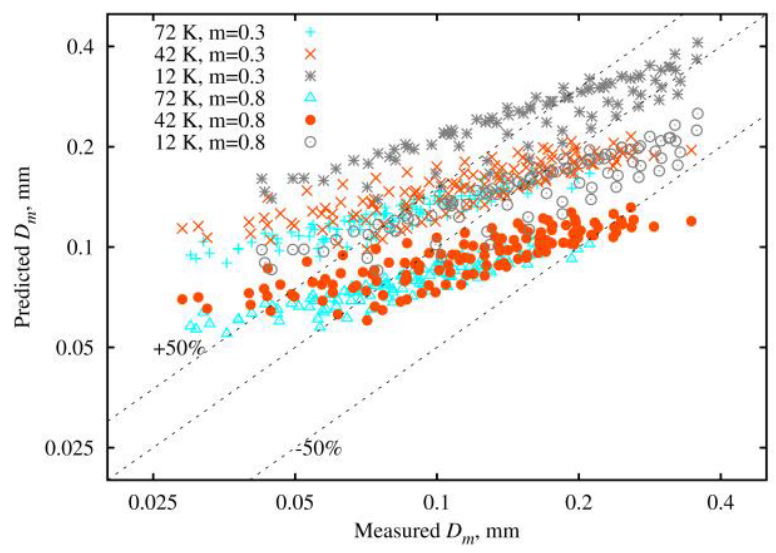

Fig. 2. Results predicted by Song's correlation against the present data on maximum diameter, obtained using initial and present $m$ values.

Another expression for the maximum diameter, given in [4] is

$$
D_{m}=1.21 a b^{-0.5}
$$

where $a=\frac{(1-m) k_{L}\left(T_{w}-T_{s}\right)}{\rho_{V} r \sqrt{\pi \alpha_{L}}}, b=\frac{m \varphi C\left(T_{s}-T_{0}\right)}{1-\rho_{V} / \rho_{L}}, k$ is the thermal conductivity, and $m$ is the fraction of the bubble surface area covered by the subcooled liquid at the temperature $T_{0}$, as proposed in [4]. Following [4], the coefficients $\varphi$ and $C$ for the present condition is equal to 1 and 65 , correspondingly. The cooling area fraction $m$ of 0.3 was indicated as the best one for approximating the experiment data in [4]. However, $m=0.8$ gives better results when applied to our data (Fig. 2). 


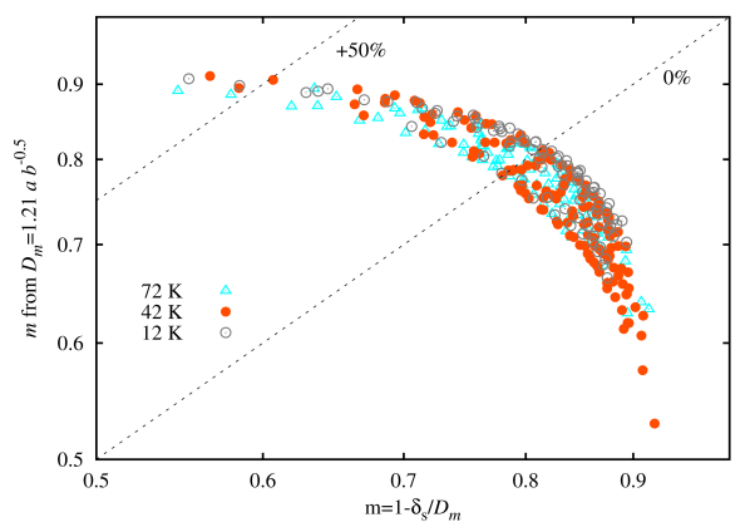

Fig. 3. Comparison of the cooled area fraction obtained from model [2] and from the numerically calculated location of the saturation temperature isotherm.

By definition, $m$ is related to the location of $T_{s}$ isotherm as follows:

$$
m=1-\delta_{s} / D_{m},
$$

where $\delta_{s}$ is the thickness of the superheated liquid layer. In the present work, $\delta_{s}$ was found numerically, together with the temperature field. Also, $m$ can be calculated by using the inverse function (3). The cooling area fractions found with the use of (3) and (4) are represented in Fig. 3; it can be readily seen that the factor $m$ loses its physical meaning when applying (3) to our experimental data. It should be noted that for our experimental conditions the temperatures between $T_{s}$ isotherm and the bubble top is bigger than $T_{0}$.

\section{Conclusions}

The present experiment data on the maximum bubble diameter was compared with the theoretical predictions by using both the initial and modified coefficient values. The approach based on the heat balance proved to be more promising for approximating both stationary and non-stationary data without major changes, because this model does not include the arbitrary coefficients. However, the physical meaning of the parameter $m$ seems to be ambiguous. The heat balance equation can be improved by using the information about the actual temperature field around the bubble.

This research was performed at the Melentiev Energy Systems Institute of the Siberian Branch of the Russian Academy of Sciences with the use of equipment of the multi-access scientific center "High Temperature Circuit".

\section{References}

1. I.C. Chu, H.C. No, C.H. Song, J. Nucl. Sci. Technol. 48936 (2011)

2. V. Prodanovic, D. Fraser, M. Salcudean, Int. J. Multiphase Flow 281 (2002)

3. J. Kim, M.H. Kim, Int. J. Multiphase Flow 321269 (2006)

4. N.H. Hoang, I.C. Chu, D.J. Euh, C.W. Song, Int. J. Heat Mass Transfer, 94174 (2016)

5. M. Colombo, M. Fairweather, Int. J. Heat Mass Transfer, 85135 (2015) 